\title{
Zerumbone promotes proliferation of endogenous neural stem cells in vascular dementia by regulating Notch signalling
}

\author{
Lei Sun ${ }^{1}$, Min $\mathrm{Li}^{2}$, Xicai Sun ${ }^{3}$, Xue $\mathrm{Li}^{4}$ \\ ${ }^{1}$ PTN Graduate Program, School of Life Sciences, Tsinghua University, Beijing 100084, China, ${ }^{2}$ Pharmaceutical Experiment Teaching \\ Center, College of Pharmacy, Harbin Medical University, Harbin 150081, China, ${ }^{3}$ Center for Stem Cell Biology and Regenerative \\ Medicine, School of Medicine, IDG/McGovern Institute for Brain Research, Tsinghua University, Beijing 100084, China, ${ }^{4}$ School \\ of Medicine, Tsinghua University, Medical Science, Beijing, China
}

\begin{abstract}
Introduction: Present investigation determines the effect of zerumbone on the proliferation of stem cells in vascular dementia (VD) rats.

Material and methods: Vascular dementia was induced by cerebral ischemia and reperfusion through non-invasive clamp. Rats were treated with zerumbone $50 \mathrm{mg} / \mathrm{kg}$ and $100 \mathrm{mg} / \mathrm{kg}$ intraperitoneally $30 \mathrm{~min}$ for four weeks after the surgery. Cognitive functions are determined by the Morris water maze (MWM) test and neurological function score in $V D$ rats. Moreover mediators of inflammation and parameters of oxidative stress were estimated in the brain tissue homogenate of ischemia-induced vascular dementia rats. The expression of proteins and $M R N A$ expressions were determined by western blot assay and RT-PCR methods. Moreover histopathological changes were observed by H\&E staining on the brain tissue of vascular dementia rats.

Results: There was a significant reduction in the cognitive function and neurological score in the zerumbone-treated group compared to the VD group of rats. Data of the study reveal that treatment with zerumbone attenuates the altered level of cytokines and markers of oxidative stress parameters in the brain tissue of VD rats. The expression of NICD, Hes-1 and Nestin proteins was significantly $(p<0.01)$ reduced in the brain tissue of the zerumbone-treated group compared to the VD group of rats. There was a significant reduction in the mRNA expression of Notch-1 and Hes-1 in the brain tissue of the zerumbone-treated group compared to the VD group of rats.

Conclusions: This study concludes that treatment with zerumbone protects the neuronal injury and ameliorates the cognitive function by stimulating the proliferation of endogenous neural stem cells. Moreover proliferation of neural stem cells was stimulated in zerumbone-treated rats by regulating the Notch signalling.
\end{abstract}

Key words: dementia, ischemia, neuronal stem cell, Notch signalling, zerumbone.

\section{Introduction}

Vascular dementia is a pathological condition of cerebral hypoperfusion and occurs due to several aetiologies including damage of vascular brain tissue [9]. Vascular dementia is one of the com- mon causes of Alzheimer's disease and dementia. In vascular dementia mainly caused due to ischemic damage of the brain and other pathogenesis, synaptic plasticity, free radical generation, neuroinflammation and excitotoxicity were observed [6]. Vascular dementia was characterized by change

\section{Communicating author}

Xue Li, PhD, School of Medicine, Tsinghua University, Medical Science, Building D120, 100084, Beijing, China, phone/fax: 0086-010-62773252, e-mail: LucasAtkinsuso@yahoo.com 
in the behaviour and reduced cognitive function. In vascular dementia, different pathological changes were observed depending on the region of the lesion. The literature reveals that cerebral ischemia affects majorly the region of brain such as striatum, hippocampus and parietal cortex to which blood was supplied by the middle cerebral artery [2]. The hippocampus region of the brain has proven to play a role for memory and the learning process and thus cerebral ischemia leads to a defect in the cognitive functions [11].

In the hippocampus and subventricular zone of the brain, proliferation of neural stem cells protects the neuronal injury [21]. In vascular dementia, cerebral injury is not able to prevent due to presence of less quantity of stem cells. It is well documented that the differentiation, proliferation and maintenance of neural stem cells was regulated by Notch signalling [8]. Notch is a transmembrane protein receptor which is activated by binding to DSL and also helps in the regulating of neurogenesis [15].

Zingiber zerumbet has traditionally been used as a medicine in China and reportedly has several medicinal properties, such as antiallergic, antioxidant, antiulcer, anti-inflammatory, anticancer and antimicrobial activities [20]. Zerumbone is a sesquiterpene isolated from rhizomes of $Z$. zerumbet [20]. Zerumbone has antitumor, antioxidant, antidiabetic, anti-nociceptive and anti-inflammatory effects $[7,16,20]$. It also ameliorates the neuropathic pain and retinal damage induced due to ischemic condition [17]. The expression of inflammatory cytokines and cyclooxygenase- 2 was inhibited by zerumbone [13]. Zerumbone also attenuates nutritional steatohepatitis by regulating gene expression related to fibrogenesis, inflammation and oxidative stress [22]. Thus, the present report determines the protective effect of Zerumbone on the neuronal injury in a vascular dementia rat model.

\section{Material and methods}

\section{Animals}

Male Sprague-Dawley rats (250-300 g) were kept under the $12 \mathrm{~h}$ of light and dark cycle in the standard condition such as $60 \pm 5 \%$ of humidity, $24 \pm 3^{\circ} \mathrm{C}$ of temperature. All the protocols of the study were approved by the institutional animal ethical committee of Tsinghua University, China (IAEC/SM-TU/2019/12).

\section{Chemicals}

Zerumbone was procured from Sigma Aldrich, USA. ELISA kits for the estimation of mediators of inflammation and parameters of oxidative stress were purchased from R\&D Systems, USA. RevertAid First Strand cDNA Synthesis kit was purchased from Thermo Scientific, USA.

\section{Experiments}

Vascular dementia was induced in the rats as per previously reported method [12]. All the rats were anesthetized by administration of chloral hydrate $(10 \% \mathrm{w} / \mathrm{v})$ intraperitoneally (i.p.) and an incision was done on the midline cervical region. Further, the vagus nerve and carotid sheath were separated out after exposing it. Ischemia was induced by a non-invasive clamp for 10 minutes and re-perfusion for 10 minutes, repeated three times. Rats were kept in the cage after suturing the incision. However, the sham group of rats were operated without inducing ischemia and reperfusion.

All the animals were divided into five different groups including the Sham group; the vascular dementia (VD) group receives a saline solution; the zerumbone $50 \mathrm{mg} / \mathrm{kg}$ and $100 \mathrm{mg} / \mathrm{kg}$ group receives zerumbone $50 \mathrm{mg} / \mathrm{kg}$ and $100 \mathrm{mg} / \mathrm{kg}$, i.p. $30 \mathrm{~min}$ after the surgery for four weeks.

\section{Determination of behavioural changes}

The Morris water maze (MWM) was used to determine behavioural changes as per a previously reported study. The water maze had a height and diameter of 40 and $100 \mathrm{~cm}$, respectively, and the platform depth was $15.5 \mathrm{~cm}$. Four quadrants were created in the apparatus with the thread and one quadrant of the platform was placed so that it could not be visualised. Swimming behaviour was monitored continuously for 6 days. Escape latency was determined as an indicator of spatial memory and the effect of zerumbone was estimated on the vascular dementia.

\section{Estimation of neurological function}

The modified neurological severity score (mNSS) was used to determine balance, touch, vision, abnormal behaviour, muscle mass, sensation and motion, as previously reported. The mNSS was calculated on a 0-18 scale, where 0 indicated normal brain func- 
tion and 18 indicated a severe deficit. Neurological function was determined at the end of protocol.

\section{Histopathological analysis}

All the rats were sacrificed by cervical dislocation and isolation of the brain was done for each rat. Brain tissue was fixed in formalin solution (10\%) and tissue was seeded into liquid paraffin. Tissue was sectioned into $8 \mu \mathrm{M}$ thickness using microtome and further section of tissue was incubated with $\mathrm{H} \& \mathrm{E}$ staining. Changes in the histopathology were determined by using a trinocular microscope.

\section{Biochemical parameters}

ELISA kits were used to determine the concentrations of inflammatory mediators, such as nuclear factor $\kappa \mathrm{B}$ (NF-KB), interleukin 6 (IL-6) and tumour necrosis factor $\alpha$ (TNF- $\alpha$ ) in the brain tissues of neuronal injury rats. Moreover, oxidative stress markers, such as malondialdehyde (MDA) and super oxide dismutase (SOD), were measured in brain tissues using ELISA kits.

\section{Western blot assay}

Brain tissues from the site of injury were separated out and the tissue was lysed in lysis buffer. The supernatant was separated by centrifuging the lysate for $5 \mathrm{~min}$ at 10,000 rpm. Sodium dodecyl sulphate-polyacrylamide gel electrophoresis was used to separate the total proteins. The separated proteins were filtered onto a nitrocellulose membrane. The membrane was incubated with Iry antibodies, including goat anti-NICD (1 : 500; Santa Cruz, USA) and anti-Hes-1 (1 : 500; Santa Cruz, USA), rabbit anti-Nestin (1 : 500; Santa Cruz, USA) and $\beta$-actin (1 : 1000; Santa Cruz, USA), overnight at $4^{\circ} \mathrm{C}$. Horseradish peroxidase-conjugated secondary antibody was incubated for $60 \mathrm{~min}$ at room temperature. Image J software (National Institutes of Health, Bethesda, MD, USA) was used to estimate band density.

\section{Real-time polymerase chain reaction (RT-PCR) analysis}

The RNA was isolated from hippocampal tissues using TRIzol reagent. The RevertAid First Strand cDNA Synthesis Kit (Fermentas, Ontario, Canada) was used to reverse-transcribe RNA. The primers mentioned below were mixed with RT2 SYBR Green
Master Mix (Superarray, Frederick, MD, USA) to determine the gene expression using Quantitative SYBR Green PCR assays.

Primer Forward Backward

Notch 1 TCG TGT GTC AAG CTG ATG AGG A GTT CGG CAG CTA CAG GTC ACA A Hes-1 GCA GAC ATT CTG GAA ATG ACT GTG A GAG TGC GCA CCT CGG TGT TA $\beta$-actin GGC TGT ATT CCC CTC CAT CG CCA GTT GGT AAC AAT GCC ATG T

\section{Statistical analysis}

All data are expressed as means \pm standard error (SEM; $n=10)$ and the statistical analysis consisted of a one-way analysis of variance (ANOVA). Post-hoc comparisons of means were carried out with Dunnett's post hoc-test using GraphPad Prism software (ver. 6.1; San Diego, CA, USA). P-values < 0.05 were considered to indicate statistical significance.

\section{Results}

\section{Zerumbone ameliorates the cognitive function}

Figure 1 shows the effect of zerumbone on the cognitive function in ischemia-induced vascular dementia rats by MWM. It was observed that the percentage of time spent in the target quadrant and number of crossing was significantly $(p<0.01)$ reduced in the VD group compared to the sham-operated group of rats. Moreover escape latency was found to be enhanced in the VD group compared to the sham group of rats. Treatment with zerumbone ameliorates the altered cognitive function in ischemia-induced vascular dementia rats.

\section{Zerumbone ameliorates neurological functions}

Vascular dementia rats treated with zerumbone on the neurological function were shown in Figure 2. In the VD group of rats, the neurological function score was enhanced compared to the sham-operated group of rats. There was a significant $(p<0.01)$ reduction in the neurological function score in the zerumbone-treated group compared to the VD group of rats.

\section{Zerumbone ameliorates the pathological changes}

The effect of zerumbone on the pathological changes in the brain tissue of ischemia-induced vascular dementia rats was shown in Figure 3. His- 
A

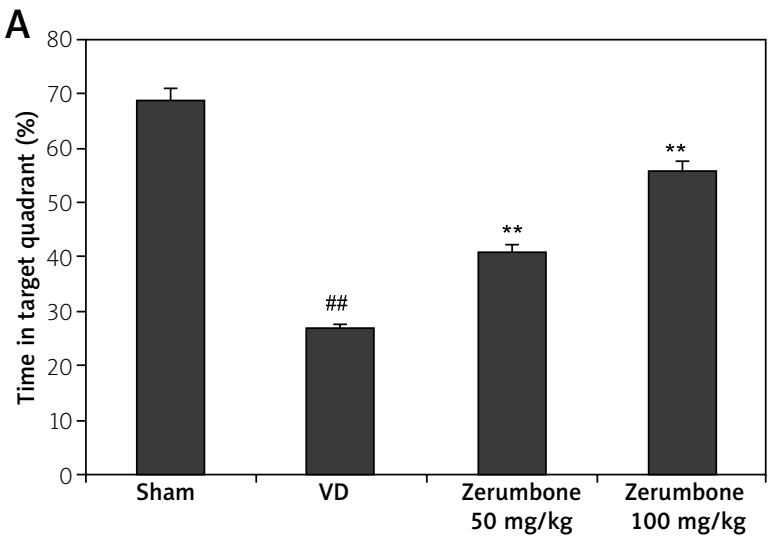

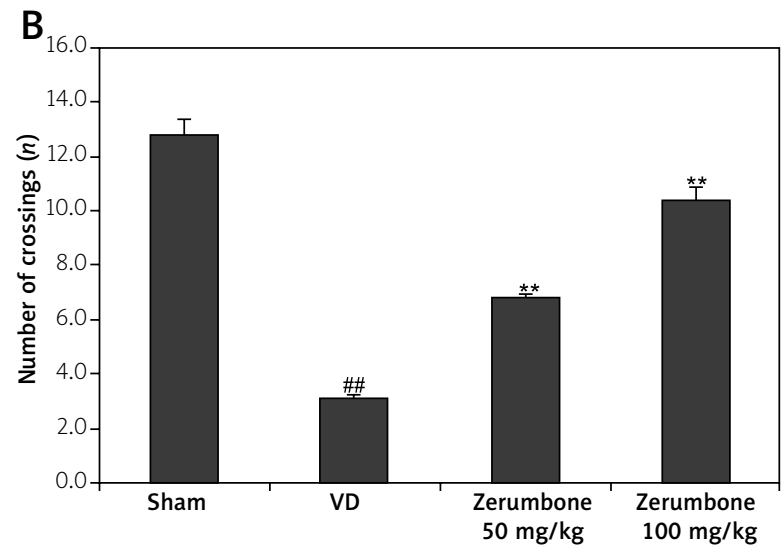

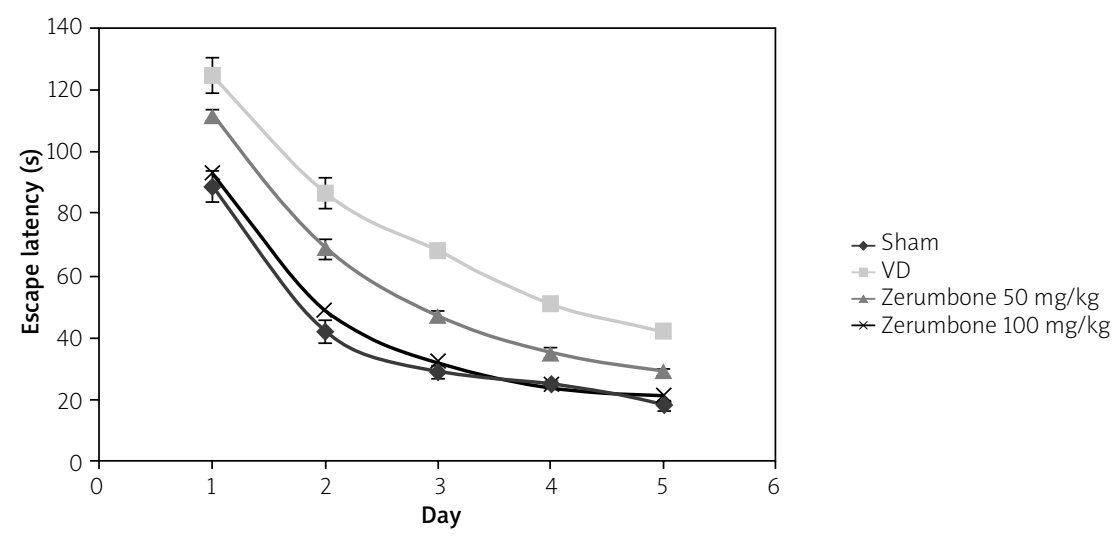

Fig. 1. Effect of zerumbone on the cognitive function in ischemia-induced vascular dementia rats, mean \pm SEM $(n=10) .{ }^{\# \#} p<0.01$ compared to the sham group, ${ }^{* *} p<0.01$ compared to the VD group.

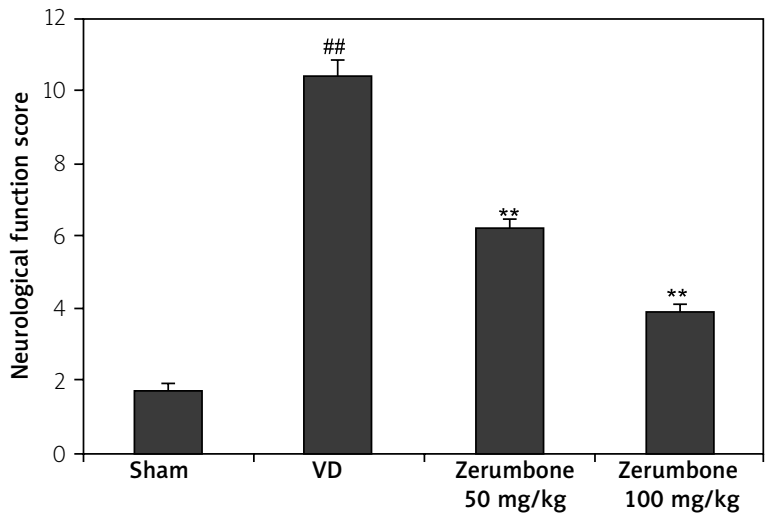

Fig. 2. Effect of zerumbone on the neurological function score in ischemia-induced vascular dementia rats, mean \pm SEM $(n=10)$. ${ }^{\# \#} p<0.01$ compared to the sham group, ${ }^{* *} p<0.01 \mathrm{com}-$ pared to the VD group. topathological changes in the brain tissue of the sham-operated group of rats shows no neuronal damage and no structural changes. However, in the VD group of rats, changes in the histopathology of brain tissue suggest the congestion of the blood cells and necrosis to neuronal cell with nucleus pyknosis and unclear architecture. Treatment with zerumbone restores the neuronal damage in brain tissue of ischemia-induced vascular dementia rats.

\section{Zerumbone ameliorates the level of mediators of inflammation}

In rats treated with zerumbone, an effect on the level of mediators of inflammation was observed in the brain tissue of ischemia-induced vascular dementia rats (Fig. 4). The level of inflammatory mediators such as NF-kB, TNF- $\alpha$ and IL- 6 in the brain 


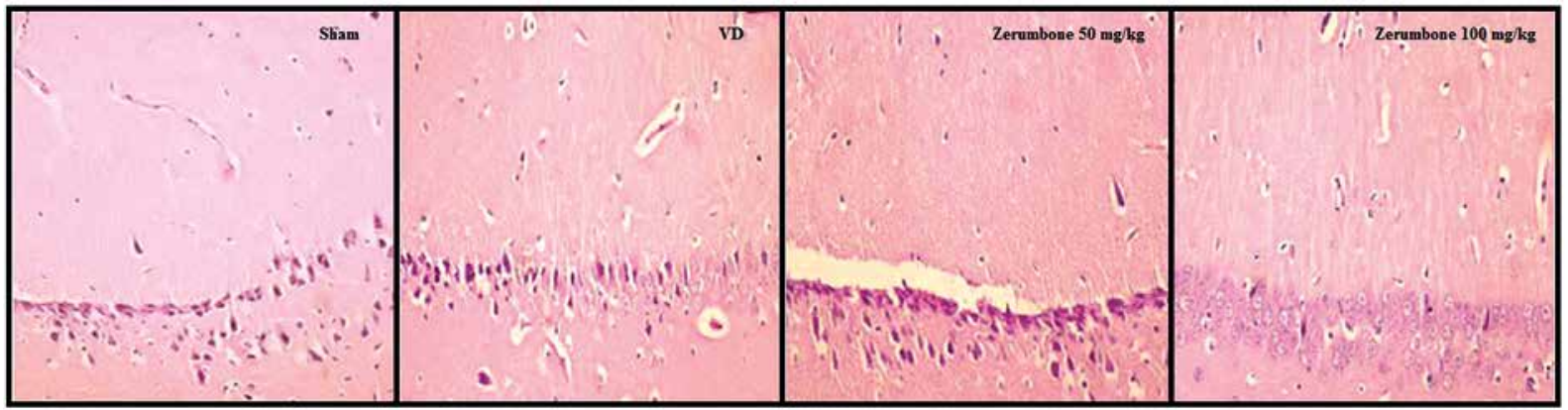

Fig. 3. Effect of zerumbone on the pathological changes in the brain tissue of ischemia-induced vascular dementia rats.

tissue homogenate was higher in the VD group than the sham-operated group of rats. There was a significant $(p<0.01)$ decrease in the level of NF-kB, TNF- $\alpha$ and IL- 6 in the brain tissue homogenate of the zerumbone-treated group compared to the VD group of rats.

\section{Zerumbone ameliorates the parameters of oxidative stress}

Parameters of oxidative stress were determined in the vascular dementia rats treated with zerumbone by ELISA. There was a significant $(p<0.01)$ increase in the level of MDA and decrease in the activity of SOD in the brain tissue homogenate of the VD group compared to the sham-operated group. In the zerumbone-treated group, the level of MDA was reduced and activity of SOD was enhanced in the

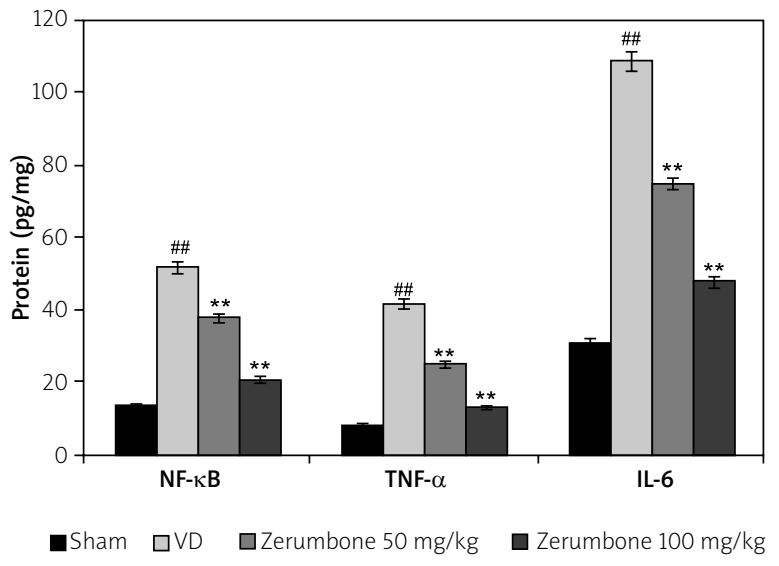

Fig. 4. Effect of zerumbone on the level of mediators of inflammation in the brain tissue of ischemia-induced vascular dementia rats, mean

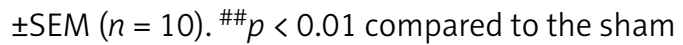
group, ${ }^{* *} p<0.01$ compared to the VD group.
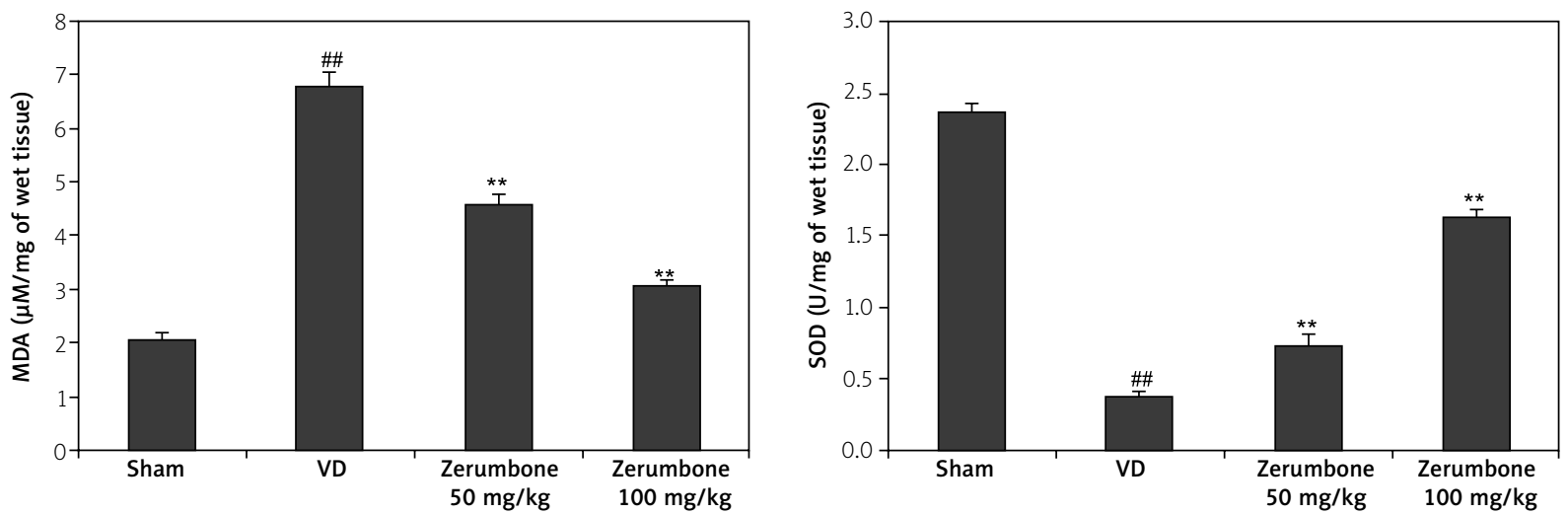

Fig. 5. Effect of zerumbone on the parameters of oxidative stress in the brain tissue of ischemia-induced vascular dementia rats, mean $\pm \operatorname{SEM}(n=10) .{ }^{\# \#} p<0.01$ compared to the sham group, ${ }^{* *} p<0.01$ compared to the VD group. 

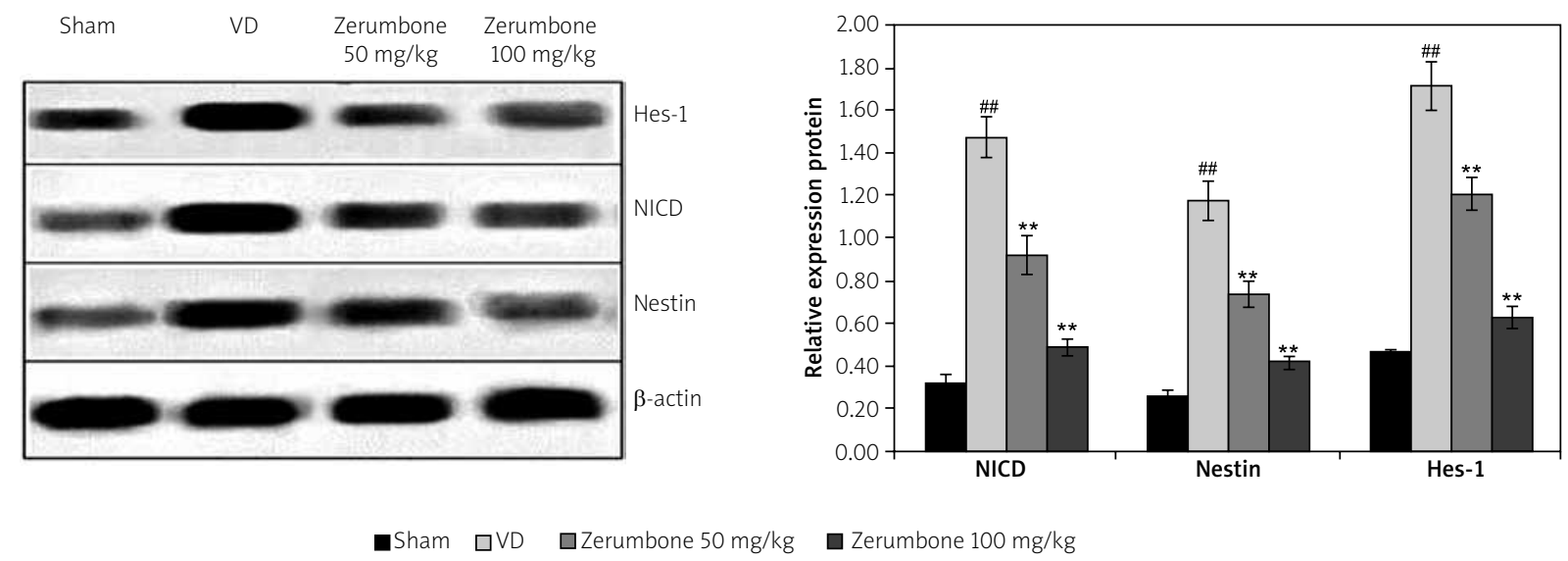

Fig. 6. Effect of zerumbone on the expression of NICD, Nestin and Hes-1 in the brain tissue of ischemia-induced vascular dementia rats, mean \pm SEM $(n=10)$. ${ }^{\# \#} p<0.01$ compared to the sham group, ${ }^{* *} p<0.01$ compared to the VD group.

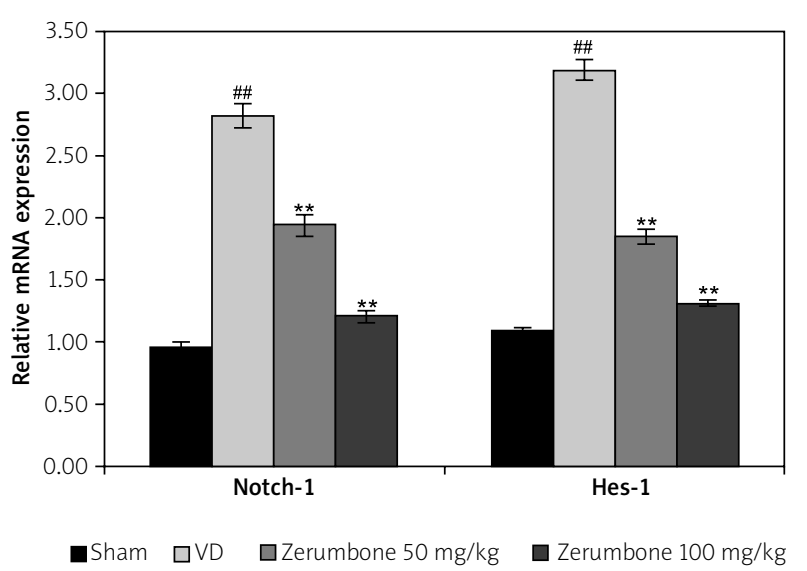

Fig. 7. Effect of zerumbone on the mRNA expression of Notch-1 and Hes-1 in the brain tissue of ischemia-induced vascular dementia rats, mean \pm SEM $(n=10) .{ }^{\# \#} p<0.01$ compared to the sham group; ${ }^{* *} p<0.01$ compared to the VD group.

brain tissue compared to in the VD group of rats in a dose dependent manner (Fig. 5).

\section{Zerumbone ameliorates the expression of NICD, Hes-1 and Nestin proteins}

Western blot assay was used for the assessment of expression of NICD, Nestin and Hes-1 proteins in the brain tissue of zerumbone-treated vascular dementia rats (Fig. 6). The expression of NICD, Nestin and Hes-1 proteins was $(p<0.01)$ enhanced in the brain tissue of the VD group compared to the sham-operated group of rats. In the zerumbone- treated group, the expression of NICD, Nestin and Hes-1 proteins in the brain tissue homogenate was reduced significantly $(p<0.01)$ compared to the VD group of rats.

\section{Zerumbone ameliorates the mRNA expression of Notch-1 and Hes-1}

The effect of zerumbone on the mRNA expression of Notch-1 and Hes-1 in the brain tissue of ischemia-induced vascular dementia rats was determined by RT-PCR method. mRNA expression of Notch-1 and Hes-1 was enhanced ( $p<0.01$ ) in the brain tissue of the VD group compared to the sham-operated group. There was a significant $(p<0.01)$ reduction in mRNA expression of Notch-1 and Hes- 1 in the brain tissue of the zerumbone-treated group compared to the VD group of rats.

\section{Discussion}

Cerebral ischemia causes neuronal injury which leads to vascular dementia and the endogenous neuronal stem cell is not able to prevent the neuronal injury [3]. There are several research studies going on the protection of brain injury and still the repair of neuronal injury is a difficult task. The literature reveals that neuronal injury is repaired by stimulating the proliferation of the endogenous neural stem cell [13]. Thus, the present investigation determines the effect of zerumbone on the proliferation of stem cells in vascular dementia rats. Cognitive functions are determined by the 
MWM test and neurological function score in VD rats. Moreover, mediators of inflammation and parameters of oxidative stress were estimated in the brain tissue homogenate of ischemia-induced vascular dementia rats. The expression of proteins and mRNA expressions were determined by western blot assay and RT-PCR methods. Moreover, histopathological changes were observed by $\mathrm{H} \& \mathrm{E}$ staining and immunohistochemistry on the brain tissue of vascular dementia rats.

Vascular dementia causes Alzheimer's disease and dementia and in both pathological conditions the patient suffers from cognitive dysfunctions. Reported studies reveal that the drug used for the management of Alzheimer's disease attenuates the cognitive function $[1,12]$. Data of this study suggest that zerumbone treatment ameliorates the learning and memory of the brain function and also significantly reduces the neurological function score compared to the VD group of rats.

There are several factors contributing to the proliferation of endogenous neural stem cells, as the enhanced level of oxidative stress and cytokines in the brain reduces the proliferation of neural stem cells. The literature reveals that treatment with zerumbone reduces the level of inflammatory cytokines and oxidative stress $[4,18]$. Results of our study suggest that zerumbone ameliorates the altered level of cytokines and parameters of oxidative stress in the brain tissue compared to the VD rats in a dose dependent manner.

Notch signalling contributes to the regulation of differentiation and proliferation of endogenous neural cells [5]. Notch is a transmembrane protein receptor, which is activated by binding to DSL and also helps in the regulating of neurogenesis [19]. The Notch intracellular domain contributes to the activation of Notch by regulating the expression of Nestin and Hes-1 protein [10] Data of this report reveals that treatment with zerumbone significantly reduces the expression of NICD, Nestin and Hes-1 protein in the brain tissue homogenate compared to the VD group of rats. RT-PCR study suggests that mRNA expression of Notch-1 and Hes- 1 was reduced in the brain tissue of the zerumbone-treated group compared to the VD group of rats. Moreover, a histopathology study reveals that treatment with zerumbone attenuates the neuronal injury in the ischemia-induced vascular dementia rats.

\section{Conclusions}

In conclusion, treatment with zerumbone protects the neuronal injury and ameliorates the cognitive function by stimulating the proliferation of endogenous neural stem cells. Moreover, proliferation of neural stem cells was stimulated in zerumbone-treated rats by regulating the Notch signalling.

\section{Author's contribution}

Lei Sun did the study design, manuscript writing; and experimental works; Min Li and Xicai Sun were responsible for the data analysis and interpretation. Xue Li contributes for the study design, literature review and guided for the conduct of the presented work.

\section{Acknowledgement}

We gratefully acknowledged the financial support from Harbin Medical University Research Innovation Fund (grant number 2016JCZX51), and also greatly appreciate the support from the technology resource sharing platform of EasyScience (www. yikexue.com) for providing essential facilities to perform the research work proposed in this paper.

\section{Disclosure}

The authors report no conflict of interest.

\section{References}

1. Baker EW, Kinder HA, West FD. Neural stem cell therapy for stroke: A multimechanistic approach to restoring neurological function. Brain Behav 2019; 9: e01214.

2. Butler TL, Kassed CA, Sanberg PR, Willing AE, Pennypacker KR. Neurodegeneration in the rat hippocampus and striatum after middle cerebral artery occlusion. Brain Res 2002; 929: 252-260.

3. Chien TY, Huang SK, Lee CJ, Tsai PW, Wang CC. Antinociceptive and anti-inflammatory effects of zerumbone against monoiodoacetate-induced arthritis. Int J Mol Sci 2016; 17: 249.

4. da Costa M, Bernardi J, Costa L, Fiuza T, Brandão R, Ribeiro MF, Amaral JD, Rodrigues CMP, Pereira ME. N-acetylcysteine treatment attenuates the cognitive impairment and synaptic plasticity loss induced by streptozotocin. Chem Biol Interact 2017; 272: 37-46.

5. De Reuck J, Maurage CA, Deramecourt V, Pasquier F, Cordonnier C, Leys D, Bordet R. Aging and cerebrovascular lesions in pure and in mixed neurodegenerative and vascular dementia brains: a neuropathological study. Folia Neuropathol 2018; 56: 81-87.

6. Enciu AM, Constantinescu SN, Popescu LM, Mureşanu DF, Popescu BO. Neurobiology of vascular dementia. J Aging Res 2011; 2011: 401604. 
7. Huang GC, Chien TY, Chen LG, Wang CC. Antitumor effects of zerumbone from Zingiber zerumbet in P-388D1 cells in vitro and in vivo. Planta Med 2005; 71: 219-224.

8. Imayoshi I, Sakamoto M, Yamaguchi M, Mori K, Kageyama R. Essential roles of Notch signaling in maintenance of neural stem cells in developing and adult brains. J Neurosci 2010; 30: 3489-3498.

9. Kalaria RN. Neuropathological diagnosis of vascular cognitive impairment and vascular dementia with implications for Alzheimer's disease. Acta Neuropathol 2016; 131: 659-685.

10. Kobayashi T, Kageyama R. Hes1 regulates embryonic stem cell differentiation by suppressing Notch signaling. Genes Cells 2010; 15: 689-698.

11. Lazarov O, Hollands C. Hippocampal neurogenesis: Learning to remember. Prog Neurobiol 2016; 138-140: 1-18.

12. Li P, Yin YL, Zhu ML, Pan GP, Zhao FR, Lu JX, Liu Z, Wang SX, Hu CP. Chronic administration of isocarbophos induces vascular cognitive impairment in rats. J Cell Mol Med 2016; 20: 731-739.

13. Liu WY, Tzeng TF, Liu IM. Zerumbone, a bioactive sesquiterpene, ameliorates diabetes-induced retinal microvascular damage through inhibition of phospho-p38 mitogen-activated protein kinase and nuclear factor-B pathways. Molecules 2016; 21: 1708.

14. Murakami A, Miyamoto M, Ohigashi H. Zerumbone, an anti-inflammatory phytochemical, induces expression of proinflammatory cytokine genes in human colon adenocarcinoma cell lines. Biofactors 2004; 21: 95-101.

15. Sato C, Zhao G, Ilagan MX. An overview of notch signaling in adult tissue renewal and maintenance. Curr Alzheimer Res 2012; 9: 227-240.

16. Sidahmed HM, Hashim NM, Abdulla MA, Ali HM, Mohan S, Abdelwahab SI, Taha MM, Fai LM, Vadivelu J. Antisecretory, gastroprotective, antioxidant and anti-Helicobcter pylori activity of zerumbone from Zingiber zerumbet (L.) Smith. PLoS One 2015; 10: e0121060.

17. Tzeng TF, Liou SS, Tzeng YC, Liu IM. Zerumbone, a phytochemical of subtropical ginger, protects against hyperglycemia-induced retinal damage in experimental diabetic rats. Nutrients 2016; 8: 449.

18. Wang X, Seekaew P, Gao X, Chen J. Traumatic brain injury stimulates neural stem cell proliferation via mammalian target of rapamycin signaling pathway activation. eNeuro 2016; 3. ENEURO.0162-16.2016.

19. Xiao MJ, Han Z, Shao B, Jin K. Notch signaling and neurogenesis in normal and stroke brain. Int I Physiol Pathophysiol Pharmacol 2009; 1: 192-202.

20. Yob NJ, Jofrry SM, Affandi MM, Teh LK, Salleh MZ, Zakaria ZA. Zingiber zerumbet (L.) Smith: a review of its ethnomedicinal, chemical, and pharmacological uses. Evid Based Complement Alternat Med 2011; 2011: 543216.

21. Young SZ, Taylor MM, Bordey A. Neurotransmitters couple brain activity to subventricular zone neurogenesis. Eur I Neurosci 2011; 33: 1123-1132.

22. Zulazmi NA, Gopalsamy B, Min JC, et al. Zerumbone alleviates neuropathic pain through the involvement of l-arginine-nitric oxide-cGMP-K+ ATP channel pathways in chronic constriction injury in mice model. Molecules 2017; 22: E555. 